\title{
SOMATOTIPOS DE ADOLESCENTES ESCOLARIZADOS DE CÓRDOBA (ARGENTINA)
}

\author{
Anahí Ruderman; Tamara Navarro; Arnaldo Mangeaud³; Vilma Cejas4 y Juan Manuel Bajo²*
}

\begin{abstract}
${ }^{1}$ Instituto Patagónico de Ciencias Sociales y Humanas-CENPAT-CONICET. Puerto Madryn. Argentina
${ }^{2}$ Cátedra de Antropología Biológica y Cultural. Facultad de Ciencias Exactas, Físicas y Naturales. Universidad Nacional de Córdoba. Córdoba. Argentina

${ }^{3}$ Cátedra de Bioestadística. Facultad de Ciencias Exactas Físicas y Naturales. Universidad Nacional de Córdoba. Córdoba. Argentina ${ }^{4}$ Escuela de Nutrición, Facultad de Ciencias Médicas. Universidad Nacional de Córdoba. Córdoba. Argentina
\end{abstract}

PALABRAS CLAVE somatotipos; auxología; adolescencia

\begin{abstract}
RESUMEN En el presente trabajo se analizan posibles respuestas diferenciales del crecimiento de los adolescentes, en particular, cambios de forma, con relación al grupo social, estimado por nivel educativo, de los padres y madres. Para cuantificar los cambios en la forma corporal se utilizó el método del somatotipo. La muestra se constituyó con 208 escolares, de ambos sexos, de 12 a 18 años pertenecientes a dos escuelas públicas de la ciudad de Córdoba, Argentina. Antes del relevamiento antropométrico, padres y alumnos respondieron una encuesta semiestructurada sobre el nivel educativo alcanzado por los primeros, y sobre sus respectivas dietas, hábitos alimentarios y actividades recreativas y físicas. En este trabajo indagamos sobre posibles asociaciones entre el somatotipo de los alumnos y el nivel educativo alcanzado por los padres. Para calcular el somatotipo fueron
\end{abstract}

relevadas variables antropométricas originales (peso, estatura, pliegue subcutáneo tricipital, subescapular, suprailíaco y de la pierna, los diámetros bicondíleos del húmero y fémur y los perímetros del brazo contraído y de la pierna). Además del somatotipo individual se obtuvo el somatotipo grupal por cada categoría de agrupamiento (sexo y edad). El grupo femenino exhibió, de acuerdo con lo esperado según la literatura, somatotipos más endomorfos que los varones. Se encontraron diferencias estadísticamente significativas en el nivel educativo entre padres y madres de las distintas escuelas. Estos resultados podrían estar influyendo en los niveles de endomorfía de los participantes, la cual fue mayor en aquellos de la escuela cuyos padres presentaron menor nivel educativo. Rev Arg Antrop Biol 19(2), 2017. doi:10.17139/ raab.2017.0019.02.05

KEY WORDS somatotype; auxology; adolescence

ABSTRACT In this study, we analyze possible differential growth responses in adolescents, in particular, changes in body shape, in relation to their parents' social group (estimated by educational level). To quantify changes in body shape the somatotype method was employed. We studied 208 schoolchildren of both sexes, 12 to 18 years old, from two public schools in the city of Córdoba, Argentina. The students and their parents filled out a semi-structured survey about educational level of the formers, diet, and physical and recreational activities. Different anthropometric variables, such as weight, height, skinfolds (triceps, subscapular,

Una herramienta valiosa que permite cuantificar y analizar la forma corporal de las personas es el somatotipo, definido como una descripción numérica de la configuración morfológica de un individuo en el momento de ser investigado (Heath y Carter, 1966, 1967; Carter 1975, 1980, 2002; Carter y Heath, 1990). Esta técnica permite clasificar a los individuos en función de los valores de los tres componentes (ectomorfo, mesomorfo, endomorfo) de su somatotipo.

El somatotipo ha sido investigado principalmente con relación al tipo de actividad física (Araujó et al., 1978; García Pérez, 1986; Classens et al., 1987; Veitia et al., 2009; Gutnik et al., 2015; Pons et al., 2015; Raković suprailiac, and leg), elbow and knee breadth, and arm and calf circumference were assessed in order to estimate the somatotype by sex and age group to estimate both individual somatotype and also by sex and age group. Female group, according to the literature, showed more endomorphic somatotypes than male group. Significant differences were found for educational level between the mothers and fathers of the different schools. These results could be influencing the participants' levels of endomorphy, which were greater in those whose parents had a lower educational level. Rev Arg Antrop Biol 19(2), 2017. doi:10.17139/raab.2017.0019.02.05

et al., 2015); a los cambios de forma y tamaño durante la niñez y la adolescencia (Rosique, 1992; Raudsepp y Jurimäe, 1996; Marrodán et al, 2001; Özener y Duyar, 2006; Ventrella et al, 2008; Irurtia Amigó et al., 2009; Mladenova et al., 2010; Lizana Arce et al., 2012) y en relación

\footnotetext{
*Correspondencia a: Juan Manuel Bajo. Arrayán 9350. 5147 Barrio Cerro Norte. Córdoba. Argentina.

E-mail: juanmabajo@gmail.com
}

Financiamiento: Secretaría de Ciencia y Técnica de la Universidad Nacional de Córdoba (SECyT-UNC).

Recibido 19 Febrero 2016; aceptado 18 Abril 2017

doi:10.17139/raab.2017.0019.02.05 
al estado nutricional y la composición corporal durante la niñez y adolescencia (Bodzsár, 1994; Bruneau-Chávez et al., 2015).

Entre los factores que afectan a la forma corporal y por ende el somatotipo durante la adolescencia se encuentra el patrón de distribución de grasa corporal que es específico de cada sexo durante el crecimiento adolescente. Un ejemplo de este dimorfismo es la tendencia en varones, por efecto de los andrógenos, hacia la disminución relativa, de la grasa corporal y el aumento de masa muscular, mientras que en las mujeres se observa una mayor acumulación de tejido adiposo principalmente en caderas y muslos, dando lugar al patrón "ginoide" o en forma de pera. Este patrón femenino se extiende hasta la menopausia, cuando decrecen los niveles de estrógeno y se reduce la actividad lipolítica sobre la adiposidad abdominal (Rebato, 2010). Pero entre estos dos tipos o patrones existe una gran diversidad que puede ser cuantificada. Así, cuando el componente que prevalece es el ectomorfo, indica un predominio de las medidas longitudinales sobre las transversales, por lo que el individuo presentará una gran superficie en relación a su volumen corporal. En cambio, cuando el componente preponderante es el mesomorfo, los individuos presentan un predominio de los componentes óseo y muscular y del tejido conjuntivo. Por último, si el componente principal es el endomorfo, entonces tales individuos tienen un predominio del tejido adiposo. El hecho de que los distintos componentes corporales estén contemplados en el cálculo del somatotipo hace que este proporcione también una certera aproximación a la composición corporal de las personas (Carter et al., 1990; Marrodán Serrano et al., 2003).

En Argentina se han realizado investigaciones sobre somatotipos y forma corporal con fines auxológicos (Bajo et al., 2009; Bajo y Mangeaud, 2010) y otras dirigidas al ámbito del deporte y la ergonomía (Beddur et al., 2004; Lentini, et al., 2004; Bejarano et al., 2005), pese a lo cual aún resta profundizar sobre los cambios morfológicos y sus posibles causas durante los procesos ontogéneticos de la pre-adolescencia y la adolescencia en poblaciones de Argentina y en especial de Córdoba. En línea con esta idea, el presente trabajo tiene la finalidad de analizar el somatotipo de una muestra de adolescentes de ambos sexos pertenecientes a dos escuelas de Córdoba y sus posibles respuestas diferenciales por sexo, edad y grupo social (nivel socioeconómico y nivel educativo).

\section{MATERIAL Y MÉTODOS}

La ciudad de Córdoba es la segunda ciudad más poblada del país y es la capital de la provincia homónima. Está ubicada en la zona centro-norte de esta provincia $\left(31^{\circ} 25^{\prime} 00^{\prime \prime} \mathrm{S}\right.$ $\left.64^{\circ} 11^{\prime} 00^{\prime \prime} \mathrm{O}\right)$. Cuenta con una población de 1.329.604 habitantes (Censo Nacional, 2010). Su participación en la actividad primaria es baja con respecto al resto de la provincia; el sector industrial, en cambio, ocupa un lugar principal en la actividad económica de la ciudad, considerándose a Córdoba como un importante centro automotriz nacional. Además, constituye uno de los centros de servicios y actividad comercial más importantes del país. A los fines de este estudio se procedió a efectuar encuestas socioeconómicas y de nivel educativo en dos escuelas de la ciudad, una ubicada en el centro y la otra en la periferia lindante con la zona rural de quintas y cultivos.

Se realizó un estudio transversal y no aleatorizado. La muestra total se conformó con 208 escolares pertenecientes a dos establecimientos educativos públicos de Córdoba: 92 alumnos del IPEM No124 (61 mujeres y 30 varones) y 116 alumnos del IPEM No268 (76 mujeres y 41 varones) cuyas edades estuvieron comprendidas entre los 12 y 18 años. El IPEM N ${ }^{\circ} 124$ es un establecimiento educativo definido como urbano marginado. Está ubicado al sureste, en la periferia de la ciudad y a él concurren también alumnos de zonas rurales aledañas. La zona pertenece al Grupo censal número 3 (González et al., 2007) que incluye a los barrios más precarios y asentamientos marginales cercanos a zonas rurales. El IPEM N 268 es un colegio urbano ubicado en el barrio céntrico de Nueva Córdoba.

Previo a la realización del estudio se obtuvieron las autorizaciones del Comité de Bioética de la provincia de Córdoba, C.I.E.I.S del Polo Sanitario y de las directoras de los establecimientos educativos. Solo fueron incluidos en la muestra aquellos alumnos cuyos padres, tutores legales o ellos mismos en el caso de ser mayores 
de edad, firmaron el consentimiento informado. El relevamiento antropométrico se realizó según las normas para investigaciones biomédicas consignadas en el protocolo de Helsinki (2008) y siguiendo los procedimientos estandarizados recomendados (Lohman et al., 1988), los cuales, para la variables relevadas en este estudio, no divergen sustancialmente de las técnicas recomendadas en el Compendio de Cineantropometría de Cabañas y Esparza (2009).

Los sujetos fueron medidos en todos los casos sobre la lateralidad corporal derecha. Las variables antropométricas medidas fueron: peso, estatura, pliegues subcutáneos tricipital, subescapular, suprailíaco y de la pierna, así como los diámetros bicondíleo del húmero y del fémur y los perímetros del brazo contraído y de la pierna. A partir de dichas variables originales, se procedió al cálculo de los componentes del somatotipo individual utilizando las fórmulas propuestas por Carter (1975) y Carter y Heath (1990):

\section{ENDOMORFIA $=0,7182+0,1451 \mathrm{x}-$ $0,00068 \mathrm{x}^{2}+0,0000014 \mathrm{x}^{3}$}

Donde $\mathrm{x}=\sum$ (pliegue tricipital, subescapular $\mathrm{y}$ suprailíaco).

MESOMORFIA $=0,858 \mathrm{U}+0,601 \mathrm{~F}+0,188 \mathrm{~B}$ $+0,161 \mathrm{P}-0,131 \mathrm{H}+4,5$

Donde $\mathrm{U}=$ diámetro bicondíleo del húmero; $\mathrm{F}=$ diámetro bicondíleo del fémur; $\mathrm{B}=$ perímetro corregido del brazo: perímetro del brazo - pliegue tricipital; $\mathrm{P}=$ perímetro corregido de la pierna: perímetro de la pierna - pliegue de la pierna; $\mathrm{H}=$ estatura.

Para obtener la ECTOMORFIA, primero se obtuvo el Índice Ponderal (IP) mediante la siguiente fórmula: $\mathrm{IP}=$ Estatura $/ \sqrt[3]{\mathrm{Peso}}$

Luego se estableció el valor de la Ectomorfía según los siguientes criterios:

Si IP $>40,75$, la ECTOMORFIA= (IP. 0,732) 28,58

Si IP $<40,75$ y $>38,28$, la ECTOMORFIA=(IP. $0,463)-17,63$

Si IP $\leq 38,28$, la ECTOMORFIA $=0,1$

Se calculó el somatotipo promedio por cada categoría de agrupamiento: sexo, edad y escue- la. Una vez obtenidos los valores promedios de cada componente del somatotipo, se obtuvo un par ordenado de valores $(\mathrm{X} ; \mathrm{Y})$ por grupo, que permitió ubicar espacialmente a los somatotipos grupales en una somatocarta:

\section{$\mathrm{X}=$ ECTOMORFIA - ENDOMORFIA \\ $\mathrm{Y}=2$. MESOMORFIA $-($ ECTOMORFIA + ENDOMORFIA)}

Para recolectar información sobre las variables ambientales (nivel educativo alcanzado y ocupación de los padres y las madres), se le entregó a cada participante un cuestionario para que fuera respondido, junto a sus padres o tutores, en el hogar. El cuestionario y la encuesta en su conjunto fueron confeccionados ad hoc por los autores de este trabajo basándose en modelos similares publicados por González Montero de Espinosa y Marrodán Serrano (2003, 2007), Agrelo (2006) y Howe et al. (2012). Los padres o tutores debían completar una cartilla en donde se les solicitaba la descripción de su empleo, trabajo o fuente de ingresos (desde desocupado, obrero manual no calificado hasta jefe, funcionario, productor rural o empresario) y por otra parte se les pedía información acerca del nivel de estudios formales alcanzado (desde primario incompleto hasta doctorado o maestría completa, con el agregado de la opción otros). En el presente trabajo hemos analizado sólo el nivel de estudios alcanzado. Para esta variable se establecieron 3 niveles, dependiendo del máximo nivel educativo alcanzado (primario; secundario o terciario/universitario) y con el fin de conocer si existían diferencias en el nivel educativo entre los padres y madres de los participantes de las distintas escuelas, se aplicó prueba de Chi-cuadrado.

Para el análisis de la información se utilizó el software SPSS versión 20 y el programa INFOSTAT 2. Para cada una de las variables métricas se obtuvieron, por sexo, edad y establecimiento educativo, el promedio y la desviación estándar.

Para determinar la significación estadística de las diferencias intra e intersexuales, se realizaron pruebas $t$ de Student o bien pruebas de Wilcoxon (U de Mann-Whitney) para dos 
muestras independientes según correspondiera (Mangeaud, 2014). Dado el pequeño tamaño muestral, particularmente reducido en varones de ciertas edades, se decidió agrupar a los integrantes de la muestra en 3 categorías de edad: en la categoría 1 se agruparon los voluntarios de 12 y 13 años de edad, en la categoría 2 los de 14 y 15 años y en la categoría 3 los de 16,17 y 18 años. Para comprobar las condiciones de aplicación necesarias se realizaron las pruebas de Shapiro-Wilks para la verificación de la hipótesis de normalidad y prueba $\mathrm{F}$ para comprobar la homogeneidad de varianzas (Mangeaud, 2014).

\section{RESULTADOS}

\section{Nivel educativo de los progenitores}

En el presente trabajo hemos utilizado solamente el nivel educativo alcanzado por los padres como indicador socioeconómico por ser una condición que se fija tempranamente y que posee cierta estabilidad temporal. Para conocer si existían diferencias en el nivel de ocupación y de estudios entre los padres y madres de los estudiantes de las escuelas analizadas, se aplicó una prueba Chi-cuadrado la cual arrojó diferencias estadísticamente significativas tanto entre los padres de las distintas escuelas $(p=0,000)$, como entre las madres $(p=0,001)$.

Los resultados que se presentan en las Tablas 1 y 2 son los que corresponden al nivel educativo de padres y madres de los alumnos participantes expresados como porcientos.

TABLA 1. Nivel educativo de los padres por escuela $y$ en porcentajes

\begin{tabular}{ccc} 
Nivel educativo & \multicolumn{2}{c}{ Escuela } \\
del padre & IPEM N ${ }^{\circ} 124$ & IPEM N²68 \\
\hline 1 & $49,4 \%$ & $22,9 \%$ \\
2 & $46,8 \%$ & $54,2 \%$ \\
3 & $3,8 \%$ & $22,9 \%$ \\
\hline
\end{tabular}

Nivel 1: primario incompleto/completo; Nivel 2: secundario incompleto/completo; Nivel 3: universitario incompleto/ completo.
TABLA 2. Nivel educativo de las madres por escuela $y$ en porcentajes

\begin{tabular}{ccc}
\hline \multirow{2}{*}{$\begin{array}{c}\text { Nivel educativo } \\
\text { de la madre }\end{array}$} & Escuela \\
\hline 1 & $41,4 \%$ & $19,8 \%$ \\
2 & $51,7 \%$ & $58,6 \%$ \\
3 & $6,9 \%$ & $21,6 \%$ \\
\hline
\end{tabular}

Nivel 1: primario incompleto/completo; Nivel 2: secundario incompleto/completo; Nivel 3: universitario incompleto/ completo.

\section{Somatotipos}

Los estadígrafos de posición y dispersión de los componentes del somatotipo de varones se presentan en la Tabla 3, así como los resultados de la comparación entre los voluntarios de las distintas escuelas. Se puede observar que los participantes del IPEM $\mathrm{N}^{\circ} 124$ mostraron valores superiores de endomorfía en todas las categorías de edad analizadas. Esta diferencia es más notable en la tercer categoría de edad $(p=0,057)$.

Por otro lado, la mesomorfía y ectomorfía no mostraron una tendencia que diferencie claramente a los varones de las distintas escuelas estudiadas.

En la Tabla 4 se observan los valores del promedio y el desvío estándar, por categoría de edad, de los componentes del somatotipo de las mujeres. También se presentan los resultados de la comparación entre escuelas. Los niveles de endomorfía fueron superiores en el grupo del IPEM N 124 en todas las categorías de edad estudiadas, con diferencias significativas en las categorías 1 y 3 ( $p=0,017$ y $p=0,001$, respectivamente). En cuanto al componente mesomorfo y ectomorfo, los valores medios fueron similares.

\section{Diferencias intersexuales}

En la Tabla 5 se observan los resultados de la comparación intersexual de los componentes del somatotipo. Para realizar esta comparación se unificó la muestra considerando varones de ambas escuelas juntos, al igual que en la muestra femenina. Las mujeres presentaron mayores 
TABLA 3. Comparación de los componentes somatotípicos entre las escuelas IPEM N²68 e IPEM 124: serie masculina

\begin{tabular}{|c|c|c|c|c|c|c|c|c|c|c|}
\hline \multirow{2}{*}{$\begin{array}{l}\text { Categoría de } \\
\text { edad }\end{array}$} & \multirow{2}{*}{ Variable } & \multicolumn{3}{|c|}{ IPEM N²68 } & \multicolumn{3}{|c|}{ IPEM N¹24 } & \multirow[b]{2}{*}{$t$} & \multirow[b]{2}{*}{$W$} & \multirow[b]{2}{*}{$p$} \\
\hline & & $\mathrm{n}$ & $\bar{x}$ & d.e. & $\mathrm{n}$ & $\bar{x}$ & d.e. & & & \\
\hline \multirow{3}{*}{1} & Endomorfia & 12 & 3,62 & 1,22 & 7 & 3,8 & 1,87 & & 69 & 0,966 \\
\hline & Mesomorfía & 12 & 4,19 & 1,01 & 7 & 4,15 & 1,09 & & 71 & 0,967 \\
\hline & Ectomorfía & 12 & 3,02 & 1,41 & 7 & 2,93 & 1,43 & 0,137 & & 0,893 \\
\hline \multirow{3}{*}{2} & Endomorfía & 14 & 3,27 & 1,52 & 16 & 4,26 & 2,15 & & 178 & 0,109 \\
\hline & Mesomorfía & 14 & 3,57 & 1,55 & 16 & 3,8 & 1,93 & & 218 & 0,966 \\
\hline & Ectomorfía & 14 & 3,5 & 1,64 & 16 & 3,02 & 1,42 & $-0,179$ & & 0,859 \\
\hline \multirow{3}{*}{3} & Endomorfía & 15 & 3,37 & 1,76 & 7 & 5,2 & 2,41 & & 108 & 0,053 \\
\hline & Mesomorfía & 15 & 4,72 & 1,35 & 7 & 4,88 & 1,59 & $-0,179$ & & 0,859 \\
\hline & Ectomorfía & 15 & 2,45 & 1,56 & 7 & 2,79 & 1,45 & & 75 & 0,723 \\
\hline
\end{tabular}

TABLA 4. Comparación de los componentes somatotípicos entre las escuelas IPEM $N^{\circ} 268$ e IPEM $N^{\circ} 124:$ serie femenina

\begin{tabular}{ccccccccccc}
\hline $\begin{array}{c}\text { Categoría de } \\
\text { edad }\end{array}$ & Variable & \multicolumn{3}{c}{ IPEM N$^{\circ} 268$} & \multicolumn{7}{c}{ IPEM N²4 } \\
& & $\mathrm{n}$ & $\overline{\mathrm{x}}$ & d.e. & $\mathrm{n}$ & $\overline{\mathrm{x}}$ & d.e. & $t$ & $W$ & $p$ \\
\hline \multirow{2}{*}{1} & Endomorfía & 23 & 4,62 & 1,26 & 19 & 5,72 & 1,56 & $-2,501$ & 0,017 \\
& Mesomorfía & 23 & 3,71 & 1,44 & 19 & 3,95 & 1,62 & & 426 & 0,658 \\
& Ectomorfía & 23 & 2,34 & 1,54 & 19 & 1,85 & 1,45 & 1,063 & 0,294 \\
\hline \multirow{2}{*}{2} & Endomorfía & 25 & 5,19 & 1,45 & 21 & 5,82 & 1,86 & $-1,289$ & 0,204 \\
& Mesomorfía & 25 & 3,91 & 1,97 & 21 & 3,24 & 1,51 & 1,264 & 0,213 \\
& Ectomorfía & 25 & 2,11 & 1,33 & 21 & 2,18 & 1,57 & $-0,168$ & 0,868 \\
\hline \multirow{2}{*}{3} & Endomorfía & 28 & 5,09 & 1,22 & 21 & 6,35 & 1,3 & $-3,454$ & 0,001 \\
& Mesomorfía & 28 & 3,36 & 1,35 & 21 & 3,87 & 1,19 & $-1,391$ & 0,171 \\
& Ectomorfía & 28 & 2,22 & 1,18 & 21 & 1,9 & 1,4 & & 447 & 0,115 \\
\hline
\end{tabular}

TABLA 5. Comparación de los componentes somatotípicos entre varones y mujeres

\begin{tabular}{ccccccccccc}
\hline \multirow{2}{*}{ Categoría de edad } & \multirow{9}{*}{ Varones } & \multicolumn{7}{c}{ Mujeres } \\
& & $\mathrm{n}$ & $\overline{\mathrm{x}}$ & d.e. & $\mathrm{n}$ & $\overline{\mathrm{x}}$ & d.e. & $t$ & $W$ & $p$ \\
\hline \multirow{3}{*}{1} & Endomorfía & 19 & 3,68 & 1,44 & 42 & 5,12 & 1,49 & & 364 & 0,001 \\
& Mesomorfía & 19 & 4,18 & 1,01 & 42 & 3,82 & 1,51 & & 699 & 0,087 \\
& Ectomorfía & 19 & 2,99 & 1,38 & 42 & 2,12 & 1,5 & & 731 & 0,027 \\
\hline \multirow{2}{*}{2} & Endomorfía & 30 & 3,80 & 1,92 & 46 & 5,48 & 1,66 & & 769 & 0,001 \\
& Mesomorfía & 30 & 3,70 & 1,74 & 46 & 3,6 & 1,79 & & 1172 & 0,857 \\
& Ectomorfía & 30 & 3,24 & 1,52 & 46 & 2,14 & 1,43 & 3,191 & & 0,002 \\
\hline \multirow{3}{*}{3} & Endomorfía & 22 & 3,95 & 2,12 & 49 & 5,63 & 1,39 & $-3,963$ & & 0,001 \\
& Mesomorfía & 22 & 4,77 & 1,81 & 49 & 3,58 & 1,30 & 3,152 & & 0,002 \\
& Ectomorfía & 22 & 2,90 & 1,52 & 49 & 1,98 & 1,20 & & 872 & 0,319 \\
\hline
\end{tabular}


niveles de endomorfía que los varones, con diferencias estadísticamente significativas en todas las categorías de edad estudiadas. El componente mesomorfo fue significativamente mayor en varones en la tercer categoría de edad $(p=0,002)$ y el componente ectomorfo mostró diferencias estadísticamente significativas a favor de los varones en las categorías 1 y 2 .

Luego de obtener las coordenadas de los somatotipos promedio se confeccionaron las somatocartas por establecimiento educativo. En ellas se puede observar que los varones del IPEM N²68 (Fig. 1) presentaron un so- matotipo predominantemente mesomorfo en todas las clases de edad. El grupo masculino de la otra escuela estudiada (Fig. 2) presentó un somatotipo promedio con predominio del endomorfismo (componente adiposo) en las categorías 2 y 3 , mientras que en la primera categoría presentaron un predominio del mesomorfismo. Las mujeres de ambas escuelas presentaron, en todas las edades estudiadas, un somatotipo predominantemente endomorfo (Figs. 1 y 2), aunque en las participantes del IPEM $\mathrm{N}^{\circ} 124$ esta tendencia fue más marcada.

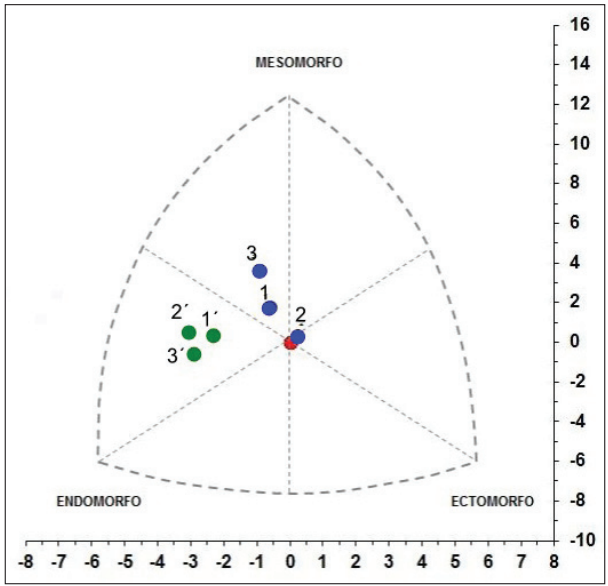

Fig. 1. Somatocarta de somatotipos promedio de varones (círculo azul) y mujeres (círculo verde) del IPEM $\mathrm{N}^{\circ} 268$, agrupados por clase de edad: 1=Clase: 112 y 13 años; 2=Clase 2:14 y 15 años-; $3=$ Clase 3:16 a 18 años.

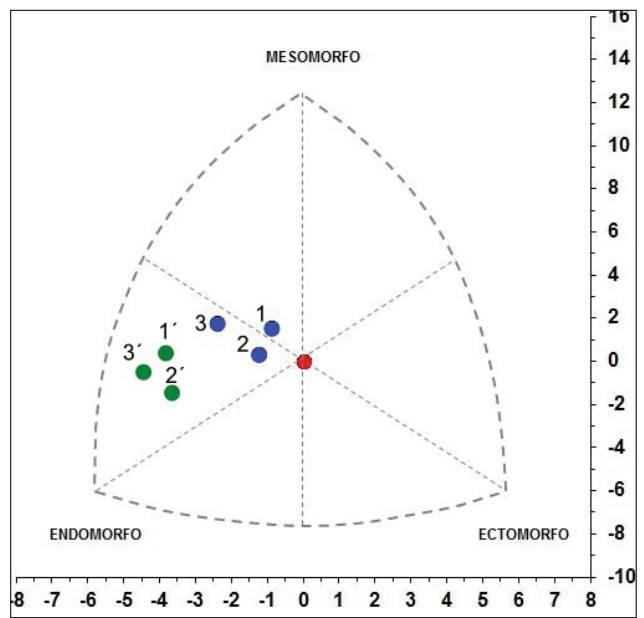

Fig. 2. Somatocarta de somatotipos promedio de varones (círculo azul) y mujeres (círculo verde) del IPEM N ${ }^{\circ} 124$, agrupados por clase de edad: 1=Clase: 112 y 13 años; 2=Clase 2:14 y 15 años-; 3=Clase 3:16 a 18 años. 


\section{DISCUSIÓN}

La variable nivel de estudios alcanzado por los progenitores ha sido utilizada en trabajos similares recientes, tal como el de Howe et al. (2012). En el presente trabajo, se observaron diferencias estadísticamente significativas en esta variable entre padres y madres de las escuelas estudiadas. Estas diferencias fueron favorables tanto a las madres como a los padres del IPEM $\mathrm{N}^{\circ} 268$, los cuales presentaron mayor escolaridad no sólo de nivel medio sino también superior. Este resultado se explicaría en parte por la mayor heterogeneidad social presente en esta escuela respecto al otro establecimiento, que por su condición de urbano marginal y pertenencia al grupo censal número 3 (González et al., 2007) muestra, por un lado, mayor vulnerabilidad social y consecuentemente por otro, una mayor homogeneidad. La escuela céntrica, IPEM $\mathrm{N}^{\circ} 268$, a diferencia de la escuela urbano marginal, IPEM $\mathrm{N}^{\circ} 124$, recibe alumnos de distintas zonas y barrios de la ciudad, los cuales son de diferentes niveles socioeconómicos. Por ejemplo, al IPEM $N^{\circ} 268$ asisten adolescentes del barrio de Nueva Córdoba (categorizado censalmente como de clase media-alta y clase alta) y otros, en mayor cantidad, de barrios humildes o villas de emergencia de zonas cercanas al centro. En el caso del IPEM N ${ }^{\circ} 124$ los alumnos en su mayoría provienen de barrio Coronel Olmedo (clase media, media baja) o bien, de villas de emergencia (villas miseria) de zonas aledañas que se ubican entre la Avenida de circunvalación y el inicio de la zona rural. La zona rural que colinda con la ciudad en su límite sud-sudeste, es una zona de producción fruti-hortícola (quintas, huertas, etc.) y ladrillales, actividades económicas primarias que empleen principalmente mano de obra infanto-juvenil. Esta situación socio-geográfica podría ser una explicación a las diferencias observadas en el nivel de escolaridad alcanzado por los padres.

Con relación al somatotipo y sus componentes, cuando se compararon escolares del mismo sexo, pertenecientes a distintas escuelas, el componente endomorfo fue el que presentó más variabilidad, mientras que la mesomorfía y la endomorfía no variaron notablemente.

El somatotipo de la muestra analizada mostró un notable dimorfismo sexual. Las diferen- cias estadísticamente significativas entre varones y mujeres en la endomorfía en todas las clases de edad estudiadas estarían explicadas, en parte, por diferencias significativas en las variables pliegue tricipital, pliegue subescapular y pliegue suprailíaco (las tres variables directamente proporcionales a la endomorfía) a favor de las mujeres. Hay que tener en cuenta también que a los 15 años los varones recientemente han experimentado el spurt puberal (Tanner, 1990) evento que determina pérdida de grasa corporal (Malina, 1974) y explica en parte por qué a esta edad las mujeres presentan más tejido graso. Esto muestra la influencia que tendría este proceso en la forma corporal de los adolescentes. Durante el período de la adolescencia los tres componentes del somatotipo, en particular la endomorfía, contribuyen a explicar las diferencias intersexuales en la morfología corporal (Rosique, 1992). La evolución de este componente en las mujeres de la muestra se caracterizó por un aumento en relación a la edad, coherentemente con Carter (1980), quien determinó que la endomorfía de las mujeres aumenta considerablemente con la edad durante la adolescencia. En estudios similares sobre adolescentes escolarizados llevados a cabo en la ciudad de Valparaíso, Chile, se pudo observar también un aumento de la endomorfia y una prevalencia actual de la misma en el sexo femenino (Lizana Arce et al., 2012). Lamentablemente, no existen muchos estudios en nuestro país o en países similares con muestras de adolescentes que posibiliten una comparación fiable con la muestra aquí estudiada. Esto se debe a que la mayor parte de los estudios sobre somatotipos están centrados en deportistas o en poblaciones adultas o niños menores que presentan alguna particularidad (deportistas, víctimas de bulling, síndrome de Down, etc.). Así, por ejemplo, los estudios realizados en Perú por Gómez Campos et al. (2012) fueron llevados a cabo en niños de 6 a 12 años y que pertenecen a poblaciones rurales asentadas en alturas moderadas (más de 2300 metros sobre el nivel del mar), por lo que presentan particularidades en su modo de crecimiento y en su composición corporal. En cambio, dos estudios similares hechos en adolescentes de escuelas rurales de la India (Subramanian et al., 2016) y en adolescentes escolarizados de la casta de los Jats en Nueva Delhi, capital de 
la India (Inuka Gakhar, 2002), al igual que los estudios realizados con adolescentes húngaros (Bodzsár y Susanne, 1999) y del país Vasco (Rebato y Rosique, 1994) indican una mayor prevalencia del componente endomórfico en las mujeres a mediados y final de la adolescencia. El patrón femenino que se observa en el presente trabajo, claramente dirigido hacia el endomorfismo, es coherente con la tendencia propia del sexo a presentar altos niveles de tejido adiposo por sobre el muscular y la linealidad corporal, y concuerda con otros estudios que señalan que, en promedio, el componente de mayor relevancia de las mujeres adolescentes es el endomorfo, seguido del mesomorfo (González Montero de Espinosa y Marrodán Serrano, 2007; Rebato y Muñoz-Cañón, 2016). Es importante señalar que las participantes del IPEM N 124 presentaron valores medios de endomorfismo mayores a

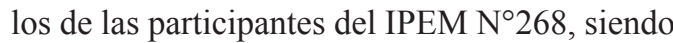
esta diferencia significativa en las clases de edad 1 y 3 . Este resultado está respaldado por diferencias significativas encontradas a esas edades en las variables originales que participan del cálculo de la endomorfía, tales como el pliegue subcutáneo tricipital, pliegue subcutáneo subescapular y pliegue subcutáneo suprailíaco. Pero que en definitiva nos hablan de una mayor homogeneidad y pobreza.En cambio, en los varones de la muestra, el componente endomórfico presentó una tendencia a disminuir conforme avanzaba la edad de los mismos. De acuerdo a esto último, Rosique (1994) propone que la adolescencia de los varones se caracteriza por una tendencia a la reducción del endomorfismo.

Los varones del IPEM $\mathrm{N}^{\circ} 124$ mostraron, al igual que sus compañeras mujeres, niveles de endomorfía promedio más elevados que los participantes del IPEM N²68. El somatotipo de los primeros mostró un predominio de la endomorfía en las clases de edad 2 y 3 , lo cual contrasta con la tendencia de los adolescentes varones a presentar un componente mesomorfo preponderante (González Montero de Espinosa y Marrodán Serrano, 2007) particularmente en torno a los 14 años, edad aproximada a la cual los varones suelen presentar un predominio de la ectomorfía y mesomorfía gracias a un crecimiento acelerado en estatura. En cambio, los escolares del IPEM N²68 mostraron un somatotipo promedio principalmente mesomorfo en todas las clases de edad estudiadas.

En la mesomorfía se observaron diferencias estadísticamente significativas en la tercera clase de edad. Los varones mostraron una tendencia al aumento de la variable con respecto a la edad mientras que en el sexo femenino la tendencia fue la opuesta. Estos resultados coinciden con los obtenidos por González Montero de Espinosa y Marrodán Serrano (2007) y están apoyados por Malina (1974) quien señala que los varones tienden a un desarrollo marcado de la mesomorfía a medida que avanza la adolescencia.

Por último, en el componente ectomórfico se observaron diferencias estadísticamente significativas en la clase de edad 2 a favor de los participantes varones, que estarían explicadas en parte por una diferencia significativa a esas edades en la variable estatura. En la primera clase de edad también se halló una diferencia estadísticamente significativa a favor de los varones en la ectomorfía, pero no se encontraron diferencias significativas ni en estatura ni en peso, ambas variables que participan del cálculo de este componente, por lo que la diferencia a esta edad en la ectomorfía podría estar siendo explicado por un efecto de interacción entre ambas variables antropométricas originales. Estos resultados, en los cuales los varones muestran una mayor ectomorfía entre las edades de 13 y 15 años, se encuentran dentro de lo esperado dado que es en torno a dichas edades que los varones experimentan un crecimiento acelerado en estatura, conduciendo a una mayor linealidad corporal final.

Se comentó anteriormente la dificultad a la hora de recabar información sobre el tipo de empleo de padres y madres, lo que redundó en una reducción considerable de los tamaños muestrales paterno y materno de las dos escuelas. Sin embargo, mediante la variable nivel educativo se logró obtener una idea aproximada pero segura del nivel socioeconómico. Se ha señalado que si los padres presentan un nivel de escolaridad adecuado, se propicia un ambiente que facilita el desarrollo propio de la adolescencia (Gutiérrez Muñiz et al., 1993; Rogers y Emmet, 2003). En general, un mayor nivel educativo da lugar a una optimización de los recursos y hábitos alimenticios, factores que pueden actuar sobre el nivel de adiposidad de los individuos (Duvigneaud et al., 2007). En este sentido, un bajo ni- 
vel educativo y cultural puede conllevar un menor interés por la salud alimentaria y la actividad física (Sobal y Stunkard, 1991) y además se corresponde con menores recursos económicos. En la muestra estudiada observamos que padres y madres de los participantes del IPEM N ${ }^{\circ} 268$ mostraron un mayor nivel educativo que los de la otra escuela participante. Estos resultados podrían estar influyendo en los somatotipos observados, en particular en los elevados valores de adiposidad revelada por los participantes del IPEM N${ }^{\circ} 124$. Aun así, es probable que otras variables no analizadas en esta oportunidad estén influyendo en estos resultados, tal como el nivel de actividad física realizada por los voluntarios.

\section{CONCLUSIONES}

Los participantes del IPEM $\mathrm{N}^{\circ} 124$ presentaron mayores niveles de endomorfía que los de la otra escuela estudiada, en ambos sexos. En particular, los varones de esta escuela mostraron un somatotipo más endomorfo que lo esperado, revelando un elevado nivel de adiposidad. Una posible explicación para esto es la influencia de variables ambientales de tipo socioeconómicas y culturales, que inciden de forma directa en la familia. Precisamente, es en el seno familiar donde de moldean los hábitos saludables, en la medida que existan recursos materiales y culturales mínimos y necesarios, que determinan la forma corporal y el crecimiento. En este sentido, los individuos con más riesgo de malnutrición por exceso de carbohidratos y grasas poli-insaturadas suelen agruparse en las clases socioeconómicas más bajas y en el medio rural. Este punto es de gran relevancia, ya que los jóvenes con sobrepeso son más proclives a transformarse en adultos obesos y a padecer más problemas de salud que los adultos con peso normal.

Por otro lado, las mujeres presentaron mayores niveles de endomorfismo que los varones, lo cual concuerda con lo esperado ya que durante la adolescencia las mujeres tienden a una mayor acumulación de tejido graso que los varones pero además este mayor endomorfismo estaría también explicado porque precisamente la subsistencia en ambientes de escasos recursos económicos se caracteriza por una dieta más pobre y monótona centrada en carbohidratos y grasas no saludables.

\section{AGRADECIMIENTOS}

A las directoras de los establecimientos educativos por la excelente predisposición. A los alumnos que voluntaria y entusiastamente participaron del estudio. A los revisores de la RAAB que con sus comentarios y sugerencias contribuyeron en mucho a mejorar este artículo.

\section{LITERATURA CITADA}

Agrelo F. 2006. Desaceleración de la talla final en adolescentes biológicamente inmaduras, que tuvieron su primer hijo a los 18 años o antes de dicha edad. Estudio epidemiológico (Tesis Doctoral). Córdoba: Editorial de la Universidad Católica de Córdoba.

Araújo C de, Gomes P, Novaes E. 1978. Somatotipo de judocas brasileiros de alto nível. Artus 1:2130.

Bajo JM, Mangeaud A. 2010. Relationship between the lung function and anthropometric measures and indexes in adolescents from Córdoba, Argentina. Am J Hum Biol 22:823-829. doi:10.1002/ ajhb. 21090

Bajo JM, Mangeaud A, Marcellino A, Cejas V, Cervilla, N. 2009. Somatotipos, función pulmonar y composición corporal en una muestra de adolescentes de la ciudad de Córdoba (Argentina). Actas de las IX Jornadas Nacionales de Antropología Biológica, Puerto Madryn. Chubut.

Beddur S, Melgartti A, Nai Aráoz J, Zanoni C, Marín G. 2004. Descripción del somatotipo de una muestra de estudiantes universitarios. Efdeportes 10(75).

Bejarano I, Dipierri J, Marrodán M, Alba J, Contreras G. 2005. Somatotipos en jóvenes amateurs del mundo andino. Rev Arg Antrop Biol 7(1):34.

Bodzsár ÉB, Pápai J. 1994. Secular trend in body proportions and composition. Human biol Budapest 25:245-254.

Bodzsár ÉB, Susanne C. 1999. Données récentes concernant les variations de somatotypes entre 7 et 18 ans au sein de la population hongroise. Bull Mém Soc Anthropol Paris 11(3-4):333-348.

Bruneau-Chávez J, España-Romero V, Lang-Tapia M, Chillón Garzón P. 2015. Diferencias en la composición corporal y somatotipo de escolares de etnia Mapuche y no Mapuche de la Comuna de Temuco-Chile. Int J Morphol 33(3):988-995. doi:10.4067/S0717-95022015000300029

Cabañas MD, Esparza F. 2009. Compendio de cineantropometría. Madrid: Editorial CTO.

Carter JEL. 1975. The Heath-Carter somatotype method. San Diego: San Diego State University.

Carter JEL. 1980. The Heath-Carter somatotype method. San Diego: San Diego State University.

Carter JEL. 2002. The Heat -Carter anthropometric somatotype. Instruction manual. San Diego: San Diego State University.

Carter JEL, Heath BH. 1990. Somatotyping: development and implications. Cambridge Studies in Biological Anthropology. Vol.5. Cambridge: Cambridge University Press. 
Censo Nacional 2010. INDEC. Censo Nacional de Población, Hogares y Viviendas 2010. http:// www.indec.gob.ar/

Claessens A, Beunen G, Wellens R, Geldof G. 1987. Somatotype and body structure of world top judoists. BJSM 27:105-113.

Duvigneaud N, Wijndaele K, Matton L, Deriemaeker P, Philippaerts R, Lefevre J, Thomis M, Duquet W. 2007. Socio-economic and lifestyle factors associated with overweight in Flemish adult men and women. BMC Public Health 7:23. doi:10.1186/1471-2458-7-23

García Pérez JG. 1986. Evaluación cineantropométrica de 101 jugadores-as cadetes de baloncesto. Estudio comparativo con una muestra aleatoria de alumnos-as de un Instituto de Formación Profesional. AMD III(11):247-252.

Gomez Campos R, JE Hespanho, M de Arruda, CP Abella, M Fargueta, Cossio-Bolanos M. 2012. Valoración del crecimiento físico por medio de la proporcionalidad corporal en escolares peruanos que viven a moderada altitud. Rev Bras Cineantropom Desempenho Hum 14(6):690-703.

González L, Peláez E, Herrero V. 2007. Vulnerabilidad social, rezago y deserción escolar en el Gran Córdoba (Argentina) 2001. IX Jornadas de Estudios de Población (AEPA). Huerta Grande, Argentina. p 31.

González Montero de Espinosa M, Marrodán Serrano MD. 2003. Crecimiento y dieta: hábitos de los jóvenes españoles. Madrid: Ed. Santa María.

González Montero de Espinosa M, Marrodán Serrano MD. 2007. La salud escolar también se mide. Guía práctica para el docente. Cuadernos de innovación. Madrid: Centro Regional de Innovación y Formación "Las Acacias".

Gutiérrez Muñiz JA, Berdasco Gómez A, Esquivel Lauzurique M, Jiménez Hernández JM, Mesa Payan D, Posada Lima E, Romero Del Sol JM, Ruben Quesada M, Ayzaguer SLC. 1993. The 1982 cuban national growth and development study: secular changes in weight and height. Acta Med Auxol 25:115-127.

Gutnik B, Zuoza A, Zuozienė I, Alekrinskis A, Nash D, Scherbina S. 2015. Body physique and dominant somatotype in elite and low-profile athletes with different specializations. Medicina 51:247-252.

Heath B, Carter JEL. 1966. A comparison of somatotype methods. Am J Phys Anthrop 24:87-100. doi:10.1002/ajpa.1330240109

Heath B, Carter JEL. 1967. A modified somatotype method. Am J Phys Anthrop 27:57-74. doi:10.1002/ajpa.1330270108

Howe L, Tilling K, Galobardes B, Smith GD, Gunnell D, Lawlor DA. 2012. Socioeconomic differences in childhood growth trajectories: at what age do height inequalities emerge? Maternal, child and adolescent health. J Epidemiol Community Health 66:143-148. doi:10.1136/ jech.2010.113068

Inuka Gakhar S. 2002. Age changes and sex differences in somatotypes among Jats of Delhi. Anthropologist Special 1:115-125.

Irurtia Amigó A, Busquets Faciabéna A, Marina Evrarda M, Pedro A, Galilea Ballarinib PA, Carrasco Marginetc M. 2009. Talla, peso, somatotipo y composición corporal en gimnastas de elite españoles desde la infancia hasta la edad adulta.
Apunts Med Esport 44(161):18-28. doi:10.1016/ S1886-6581(09)70104-5

Lentini NA, Cardey ML, Aquilino G, Dolce PG. 2004. Estudio somatotípico en deportistas de alto rendimiento de Argentina. AMD 21(104):497-509.

Lizana Arce P, Almagiá Flores A, Simpson Lelievre C, Ivanovic Marincovic D, Binvignat Gutiérrez O, Berral de la Rosa F. 2012. Changes of somatotype in high school students, $\mathrm{V}$ region, Chile: 1985-2010. Nutr Hosp 27(1):270-275.

Lohman T, Roche A, Martorell R. 1988. Anthropometric standardization reference manual. Ed. Human Kinetics Books, Illinois.

Malina R. 1974. Adolescent changes in size, build, composition and performance. Hum Biol 46(1):117-131.

Mangeaud A. 2014. Bioestadística I. Córdoba: Ed Ferreyra.

Marrodán MD, Aréchiga J, Moreno-Romero S. 2001. Cambios somatotípicos durante el crecimiento en población mexicana masculina. Antropo 1:43-50.

Marrodán MD, González Montero de Espinosa M, Prado C. 2003. Antropología de la nutrición: técnicas, métodos y aplicaciones. Madrid: Editorial Noesis.

Mladenova S, Nikolova M, Andreenko E, Boyadjiev D. 2010. Somatotypological characterization of bulgarian children and adolescents (Smolyan Region). Coll Antropol 34(3):963-971.

Özener B, Duyar I. 2008. The effect of labour on somatotype of males during the adolescent growth period. HOMO 59(2): 161-172. doi:10.1016/j. jchb.2007.12.002

Pons V, Riera J, Galilea PA, Drobnic F, Banquells M, Ruiz O. 2015. Características antropométricas, composición corporal y somatotipo por deportes. Datos de referencia del CAR de San Sugat, 19892013. Apunts Med Esport 50(186):65-72. http:// dx.doi.org/10.1016/j.apunts.2015.01.002

Raković A, Savanović V, Stanković D, Pavlović R, Simeonov A, Petković E. 2015. Analysis of the elite athlete's somatotypes. Acta Kinesiol 9 (Suppl.1):47-53.

Raudsepp L, Jürimäe T. 1996. Somatotype and physical fitness of prepubertal children. Coll Antropol 20(1):53-59.

Rebato E. 2010. Crecimiento: una visión desde la antropología física. Rev Esp Antrop Fís 31:111-122.

Rebato E, Rosique J. 1994. Estudio del somatotipo en la comarca de Busturia. Cuad Secc Antrop 12:1177.

Rebato E, Muñoz-Cañón M. 2016. Influencia del estatus socioeconómico (SES) sobre la variabilidad antropométrica en estudiantes universitarios del país Vasco (España). Rev Arg Antropol Biol 18(1):1-11. doi:10.17139/raab.2016.0018.01.02

Rogers I, Emmett P. 2003. The effect of maternal smoking status, educational level and age on food and nutrient intakes in preschool children: results from the Avon longitudinal study of parents and children. Eur J Clin Nutr 57:854-864. doi:10.1038/sj.ejen.1601619

Rosique J. 1992. Estudio transversal del crecimiento en escolares vizcainos. La variación antropométrica como componente de la estructura biológica de la población. Tesis Doctoral. Universidad del País Vasco. UPV-EHU.

Sobal J, Stunkard AJ. 1991. Socioeconomic status 
and obesity: a review of the literature. Pshychol Bull 105:260-275.

Subramanian S K, Vivek Kumar S, Vinayathan A, Krishnakumar R, Rajendran R. 2016. Somatotyping in adolescents: stratified by sex and physical activity. Int J Anat Appl Physiol. 2(3):32-38.

Tanner JM. 1990. Growth as a mirror of conditions in society. Estocolmo: GM Lindgren.

Veitia W, Hernández I, Leon Pérez S, Echevarría
García I. 2009. El somatotipo de la voleivolista de alto nivel de actuación: período 19922000. Apunts Med Esport 44(163):127-132. doi:10.1016/S1886-6581(09)70120-3

Ventrella AR, Semprolia S, Jürimäeb J,Tosellia S, Claessensc AL, Jürimäeb T, Brasilia P. 2008. Somatotype in 6-11-year-old Italian and Estonian schoolchildren. HOMO 59(5):383-396. doi:10.1016/j.jchb.2007.07.001 